\title{
Ceratectomia fotorrefrativa para correção da baixa miopia utilizando diferentes perfis de ablação
}

Photorefractive Keratectomy for Low myopia correction using different ablation profiles

$\begin{array}{lrrrr}\text { Paulo } & \text { Dantas } & \text { Rodrigues }^{1} & \\ \text { Mauro } & \text { Nisfii }^{2} & & & \\ \text { Mauro } & \text { Silveira } & \text { de } & \text { Queiroz } & \text { Campos }^{3} \\ \text { Wallace } & \text { Cframon }^{4} & & \end{array}$

\section{RES UMO}

Objetivos: O presente estudo prospectivo compara os resultados obtidos pela ceratectomia fotorrefrativa com uma ou duas zonas de ablação para tratamento de baixa miopia. Métodos: Dezenove pacientes com graus de miopia até 6,00 dioptrias foram submetidos a PRK com diferentes perfis de ablação em cada olho. Foi realizado tratamento esférico com uma única zona de ablação de $6,0 \mathrm{~mm}$, ao passo que o outro olho recebeu tratamento com duas zonas de ablação, sendo uma de $6,0 \mathrm{~mm}$ e outra de $6,5 \mathrm{~mm}$. Todos os tratamentos foram programados para correção total do equivalente esférico. Resultados: No grupo multizona (MZ), o equivalente esférico pré-operatório foi em média de $-2,87$ dioptrias $(-1,25$ a -4,62D). No grupo submetido à cirurgia com uma única zona de ablação (ZU), o equivalente esférico pré-operatório foi de $-2,78$ dioptrias (-1,37 a -4,62 D). Não foi observada diferença significante entre os resultados obtidos nos dois grupos com relação ao equivalente esférico final $(\mathrm{p}=0,391)$. No primeiro mês de pósoperatório, 84\% dos pacientes do grupo MZ (múltiplas zonas de ablação) e 95\% do grupo ZU (única zona de ablação), apresentaram acuidade visual sem correção melhor ou igual a 20/40. Com relação ao tempo transcorrido até se atingir a melhor acuidade visual, não houve diferença significante entre os dois grupos $(p=0,53)$. Conclusão: Os resultados obtidos utilizando-se diferentes perfis de ablação para tratamento de baixas miopias foram equivalentes no que diz respeito a equivalente esférico final, indução de astigmatismo e incidência de complicações. O estudo conclui que não foi possível observar diferença estatisticamente significante entre os dois grupos, podendo-se utilizar qualquer um dos procedimentos no tratamento das baixas miopias.

Descritores: Ceratectomia fotorrefrativa pro excimer laser; Miopia/cirurgia

\begin{tabular}{l}
\hline IN T R O D UÇ̃o \\
\hline A ceratectomia fotorrefrativa (do inglês, Photorefractive Keratectomy, \\
PRK) permite produzir um aplanamento da região central da córnea, corri- \\
gindo desta forma a miopia. As complicações associadas a PRK podem \\
estar associadas ao perfil da fotoablação corneana (profundidade, diâmetro \\
e transição) e incluem a regressão do tratamento, imprevisibilidade da \\
ametropia tratada, cicatrização com formação de opacidades de padrão \\
reticular (haze), e deslumbramento ou visão de halos ${ }^{(1)}$. A fim de minimizar \\
estes efeitos indesejáveis houve o desenvolvimento de variações na técni-
\end{tabular} Medicina (UNIFESP-EPM).

${ }^{2}$ Professor de Pós-Graduação, Departamento de Oftalmologia, Universidade Federal de São Paulo - Escola Paulista de Medicina (UNIFESP-EPM).

${ }^{3}$ Livre Docente do Departamento de Oftalmologia, Universidade Federal de São Paulo - Escola Paulista de Medicina (UNIFESP-EPM).

${ }^{4}$ Pesquisador Associado, Departamento de Oftalmologia, Universidade Federal de São Paulo - Escola Paulista de Medicina (UNIFESP-EPM).

Endereço para correspondência: Rua Viaza, 536 - São Paulo(SP)04633-050.E-mail:pdantas@oftalmo.com.br

xcimer laser; Miopia/cirurgia 
ca cirúrgica da PRK, como a criação do perfil de múltiplas zonas ópticas que cria uma transição mais gradual entre a área tratada e a não tratada.

Inicialmente idealizada para correção da alta miopia, a técnica de múltiplas zonas será estudada comparativamente à utilização de zona única, com relação à incidência de complicações e recuperação visual em pacientes com baixa miopia.

\section{MÉTODOS}

\section{Seleção de pacientes}

O presente estudo utilizou a metodologia de um ensaio clínico, sendo analítico, prospectivo e randomizado, para a avaliação do efeito de 2 intervenções de ceratectomia fotorrefrativa para correção da miopia de 38 olhos de 19 pacientes.

Os pacientes foram selecionados após demanda espontânea ao ambulatório do Serviço de Cirurgia Refrativa do Departamento de Oftalmologia da Universidade Federal de São Paulo - Escola Paulista de Medicina (UNIFESP - EPM), de forma seqüencial. A participação no estudo foi proposta a todos os pacientes com graus de miopia em ambos olhos de até $-6,00$ dioptrias (equivalente esférico), maiores de 21 anos, do sexo masculino e feminino, e que não possuíssem qualquer contra-indicação ocular ou sistêmica à realização do procedimento. Pacientes com mais de 1,00 dioptria de astigmatismo foram excluídos. Todos os pacientes foram submetidos a PRK com diferentes perfis de ablação em cada olho. De forma aleatória, um olho foi submetido à ablação utilizando-se uma zona óptica única de perfil esférico de 6,0 $\mathrm{mm}$ (grupo ZU), e o outro olho recebeu uma ablação com duas zonas ópticas, sendo uma de $6,0 \mathrm{~mm}$ de perfil esférico, para qual foi programado $100 \%$ do tratamento, e outra anelar, utilizada como transição à área não tratada, medindo de 6,0 a $6,5 \mathrm{~mm}$ de diâmetro onde se efetuou $50 \%$ da ametropia tratada (grupo MZ). Ambos os padrões de tratamento foram feitos de forma ininterrupta e contínua (tratamento de uma passagem - "single pass"). As cirurgias foram realizadas com intervalo aproximado de 3 semanas.

Os pacientes foram devidamente orientados quanto à segurança e à eficácia do procedimento, assim como as possíveis complicações. Todos assinaram termo de consentimento segundo protocolo aprovado pela Comissão de Ética do Hospital São Paulo e Universidade Federal de São Paulo.

\section{Exame oftalmológico}

A avaliação oftalmológica inicial consistiu em: medida da acuidade visual sem correção, com a correção utilizada pelo paciente, e com a melhor correção obtida por óculos, além de videoceratografia computadorizada (EyeSys ${ }^{\circledR}$ System 2000), refratometria estática (após instilação de cloridrato de ciclopentolato $1 \%$ duas gotas com intervalo de 3 minutos), exame biomicroscópico, oftalmoscopia binocular indireta e tonometria de aplanação.

\section{Seguimento}

Os pacientes foram avaliados no pré-operatório, $7^{\circ}, 15^{\circ}$ dias, $1,3,6$ e 10 meses de pós-operatório. No $7^{\circ}$ e $15^{\circ}$ dias foram avaliadas a reepitelização e tonometria, nas outras visitas exame oftalmológico completo foi realizado.

A videoceratografia pré-operatória e a obtida em cada retorno do paciente a partir do terceiro mês, foram utilizadas para impressão do mapa diferencial, com a finalidade de identificar padrões topográficos. O padrão denominado ilha central foi definido como sendo uma área central mais curva, com pelo menos 3 dioptrias de diferença com a região ao seu redor, adjacente e medindo pelo menos 1,0 $\mathrm{mm}$ de diâmetro.

A quantificação da opacidade corneana resultante do processo de cicatrização foi realizada conforme a escala abaixo ${ }^{(2)}$.

zero: ausência de opacidade

1: opacidade visível apenas com retro-iluminação

2: opacidade visível com iluminação direta ou fenda

3: opacidade que obscurece pouco os detalhes irianos

4: opacidade causando grande obscurecimento de detalhes da íris.

\section{Procedimento cirúrgico}

Todas as cirurgias foram realizadas no Excimer laser Summit SVS Apex plus ${ }^{\circledR}$, pelos estagiários do setor de Cirurgia Refrativa.

Após posicionamento e alinhamento adequados, foi delimitada a área a ser desepitelizada com marcador de $6,5 \mathrm{~mm}$ e a retirada mecânica do epitélio corneano com espátula realizada de forma rápida e eficaz, a fim de se evitar a desidratação excessiva do estroma corneano.

Todos os tratamentos foram programados para a correção total do equivalente esférico, baseados na refratometria estática do paciente. Imediatamente após o término da cirurgia, uma lente de contato descartável foi colocada.

\section{Análise estatística}

$\mathrm{Na}$ análise estatística, os dados foram considerados pareados por se tratarem de informações coletadas de ambos os olhos do mesmo paciente, que foram submetidos à ablação com laser com perfil MZ ou ZU. A análise estatística foi realizada por meio de testes paramétricos para as médias ( $\mathrm{t}$ de Student) para a análise das medidas refracionais e astigmatismo. O teste do qui-quadrado foi realizado para comparar os dois grupos quanto à acuidade visual na última visita e o tempo transcorrido até atingir a melhor visão. Foram determinados intervalos de confiança de $95 \%$.

\section{RESULTADOS}

\section{Pacientes}

A amostra foi composta por 38 olhos de 19 pacientes, sendo 10 do sexo feminino e 9 do sexo masculino, com idades 
variando entre 23 e 43 anos (média de 32,5 anos; mediana de 32 anos). O período de seguimento variou de 6 a 12 meses (média de 9,4 meses).

\section{Equivalente esférico}

No grupo multizona (MZ), o equivalente esférico pré-operatório médio foi de $-2,87 \mathrm{D}$ (variando de $-1,25 \mathrm{a}-4,62 \mathrm{D}$ ). No grupo submetido à cirurgia com uma única zona de ablação (ZU), o equivalente esférico pré-operatório foi de $-2,78 \mathrm{D}$ (variando de $-1,37$ a $-4,62 \mathrm{D}$ ).

A tabela 1 contém as médias do equivalente esférico no decorrer do seguimento, para os dois perfis de ablação utilizados.

Não foi observada diferença significante entre os resultados obtidos nos dois grupos com relação ao equivalente esférico obtido na visita mais recente $(p=0,391)$, assim como no primeiro, terceiro e sexto meses de pós-operatório $(\mathrm{p}>0,05)$.

O gráfico 1 representa o equivalente esférico pré-operatório e na visita pós-operatória mais recente, para cada paciente envolvido no estudo.

\section{Acuidade visual}

A evolução da acuidade visual sem correção nos dois grupos pode ser observada nas tabelas 2 e 3 .

\begin{tabular}{|c|c|c|c|}
\hline \multicolumn{4}{|c|}{ Equivalente Esférico (dioptrias) } \\
\hline Tempo Seguimento & Múltiplas zonas & Zona única & \\
\hline Pré-Operatório & $-2,87 \pm 0,26$ & $-2,78 \pm 0,26$ & \\
\hline 1 mês & $0,15 \pm 0,47$ & $0,39 \pm 0,70$ & $p>0,05$ \\
\hline 3 meses & $0,07 \pm 0,48$ & $0,10 \pm 0,29$ & $p>0,05$ \\
\hline 6 meses & $0,01 \pm 0,33$ & $0,05 \pm 0,27$ & $p>0,05$ \\
\hline Visita mais recente & $0,13 \pm 0,31$ & $0,02 \pm 0,27$ & $p>0,05$ \\
\hline
\end{tabular}

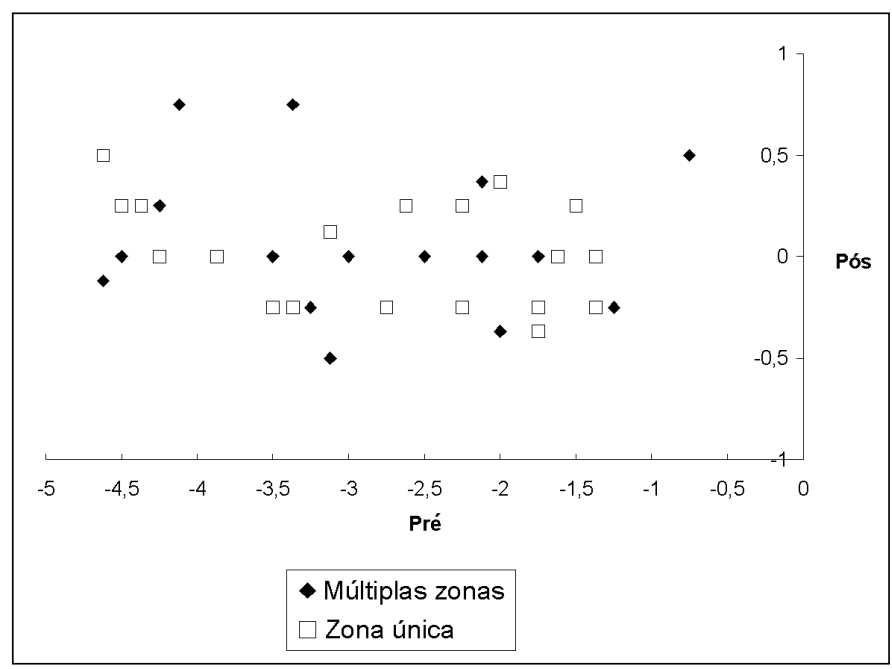

Gráfico 1 - Equivalente esférico pré-operatório e pós-operatório (última visita)

\begin{tabular}{|c|c|c|c|c|}
\hline Acuidade visual & 1 mês & 3 meses & 6 meses & Última visita \\
\hline $20 / 15-20 / 20$ & 4 & 11 & 10 & 10 \\
\hline $20 / 25-20 / 30$ & 7 & 6 & 9 & 9 \\
\hline $20 / 40-20 / 80$ & 8 & 2 & 0 & 0 \\
\hline pior $20 / 80$ & 0 & 0 & 0 & 0 \\
\hline
\end{tabular}

Tabela 3. Acuidade visual sem correção nos diferentes períodos de seguimento no grupo submetido a fotoceratectomia com única zona (ZU)

\begin{tabular}{lcccc} 
Acuidade visual & $\mathbf{1}$ mês & $\mathbf{3}$ meses & $\mathbf{6}$ meses & Última visita \\
$20 / 15-20 / 20$ & 8 & 12 & 15 & 16 \\
$20 / 25-20 / 30$ & 7 & 5 & 4 & 3 \\
$20 / 40-20 / 80$ & 4 & 1 & 0 & 0 \\
pior $20 / 80$ & 0 & 0 & 0 & 0 \\
\hline
\end{tabular}

Com relação ao tempo transcorrido até atingir a melhor acuidade visual sem correção, não houve diferença significante entre os dois grupos $(\mathrm{p}=0,53)$. Considerando-se a acuidade visual na visita mais recente, o grupo ZU apresentou uma tendência a melhor acuidade visual sem correção do que o grupo $\mathrm{MZ}(\mathrm{p}=0,036)$. Não houve diferença significante entre a acuidade visual nos dois grupos, nos pós-operatórios de 1 mês ( $p=0,30), 3$ meses $(p=0,58)$ e 6 meses $(p=0,17)$.

No grupo MZ, dois olhos apresentaram perda de uma linha da acuidade visual corrigida com óculos, sendo que em ambos, a $A V$ passou de $20 / 20$ para 20/25. No grupo ZU, não foi observada perda de linha no pós-operatório de 12 meses. Um olho do grupo MZ apresentou ganho de 1 ou mais linhas na acuidade visual.

O paciente $\mathrm{n}^{\circ} 9$ apresentava acuidade visual no olho esquerdo (grupo MZ) de 20/40 com correção (equivalente esférico $-4,25$ dioptrias) e evoluiu após tratamento com acuidade visual de 20/30. Os demais pacientes apresentavam 20/20 com correção no pré-operatório.

\section{Videoceratografia computadorizada}

Foi possível obter a videoceratografia em ambos olhos de 12 pacientes, portanto em 12 olhos de cada grupo. A análise diferencial não mostrou presença de ilha central em qualquer dos dois grupos em nenhuma visita.

\section{Opacidade corneana}

$\mathrm{Na}$ visita mais recente foi observada a presença de "haze" em 2 olhos no grupo MZ e em 3 olhos no grupo ZU. Um paciente apresentou "haze" grau I em ambos olhos que foi notado no $12^{\circ}$ mês. Em outro olho, observou-se "haze" transitório no $3^{\circ}$ mês de pós-operatório, que não foi observado na visita mais recente.

Em 1 olho do grupo MZ observou-se presença de "haze" grau II, localizado inferiormente, e que surgiu no $12^{\circ}$ mês de 
pós-operatório. O outro olho (paciente com "haze" bilateral) foi classificado como grau I. Nos 3 olhos do grupo ZU, que apresentaram "haze" na visita mais recente, este foi grau I.

\section{Astigmatismo}

$\mathrm{O}$ astigmatismo pré-operatório em ambos grupos situavase entre zero e $0,75 \mathrm{D}$.

Não foi observada diferença significante entre o astigmatismo final dos dois grupos no pós-operatório $(\mathrm{p}=0,65)$.

\section{DISCUSSÃO}

Os estudos iniciais envolvendo a ceratectomia fotorrefrativa demonstraram segurança, previsibilidade ${ }^{(3)}$ e estabilida$\mathrm{de}^{(1,4)}$ do procedimento para o tratamento de miopias até 6 dioptrias. O tratamento de miopias altas não apresentou resultados tão satisfatórios, sendo acompanhado de uma maior incidência de "haze" e regressão. A necessidade de zonas ópticas menores contribui para o aparecimento de queixas de ofuscamento e percepção de halos ${ }^{(3)}$. Observa-se também uma maior incidência de perda de linhas de acuidade visual que seria atribuída por alguns autores a espessamento e irregularidade epitelial ${ }^{(5)}$.

A menor experiência com tratamento das altas miopias levou ao desenvolvimento de uma série de pequenas variações na técnica cirúrgica, com o objetivo de melhorar o resultado final. Buscava-se um procedimento que utilizasse uma zona de ablação grande o suficiente para evitar halos, reduzisse a quantidade de tecido removida, deixasse uma superfície mais regular, com uma zona de transição gradual entre a córnea tratada e a não tratada. Surgiu a técnica de múltiplas zonas ópticas, inicialmente realizada através de múltiplas passagens (multipass). A evolução dos aparelhos permitiu que se realizasse o tratamento com múltiplas zonas em uma única passagem (singlepass), com a vantagem de ser mais rápido e mais preciso com relação à centralização do tratamento ${ }^{(6)}$.

Em estudo realizado em 75 olhos, com miopia entre -1,50 D e -10,00 D, comparando diferentes perfis de ablação, foi observada menos hipermetropia no primeiro mês em olhos tratados com três zonas de ablação (multizona), e uma recuperação visual mais rápida ${ }^{(6)}$.

Em contrapartida, outro estudo aonde pacientes submetidos a tratamentos de $-6,00 \mathrm{D}$ foram divididos em 3 grupos: zona de ablação de $5,0 \mathrm{~mm}$; zona de ablação de $6,0 \mathrm{~mm}$ e multizona $(5,0$ e $6,0 \mathrm{~mm})$ mostrou que aqueles submetidos a tratamento com $6,0 \mathrm{~mm}$, apresentaram resultado mais previsível e menos hipermetropia no primeiro mês. Convém ressaltar, no entanto, que os tratamentos com duas zonas de ablação foram realizados em duas passagens, necessitando interrupção do tratamento para reprogramar o laser ${ }^{(7)}$.

\section{Equivalente esférico}

A evolução da média do equivalente esférico nos dois grupos ocorreu de forma semelhante, mantendo-se entre $+0,50$ e - 0,50 dioptrias em $100 \%$ dos casos. Em trabalho já referido, não se observou diferença estatisticamente significante entre os grupos que utilizaram diferentes perfis de ablação, no que se refere ao equivalente esférico no sexto mês pós-operatório. Entre os pacientes tratados com única zona de ablação, $80 \%$ apresentava equivalente esférico entre $+0,50$ e $-0,50$ dioptrias no sexto mês, enquanto $88 \%$ daqueles tratados com múltiplas zonas situava-se nesta faixa ${ }^{(6)}$.

\section{Acuidade Visual}

Considerando-se o perfil dos pacientes candidatos a correção cirúrgica da miopia, torna-se importante avaliar o tempo de recuperação visual. No terceiro mês de pós-operatório, 12 olhos do grupo $\mathrm{ZU}$ e 13 do grupo MZ haviam atingido a acuidade visual final sem correção. Neste estudo não se observou diferença estatisticamente significante entre os dois grupos.

A acuidade visual no período pós-operatório precoce relaciona-se principalmente com a regularidade do epitélio corneano, a hipermetropia inicial e o padrão topográfico. Já foi observada recuperação visual mais rápida em pacientes tratados com múltiplas zonas ${ }^{(6)}$. No presente estudo, $42 \%(8 / 19)$ no grupo MZ e 68\% (13/19) no grupo ZU apresentaram acuidade visual melhor ou igual a 20/25 no primeiro mês de pósoperatório.

A acuidade visual sem correção não apresentou diferença estatisticamente significante entre os dois grupos até o sexto mês de pós-operatório. Na última visita, observou-se uma tendência a um melhor resultado no grupo ZU. Esta observação poderia ser mais valorizada se dispuséssemos de uma amostra maior. Esta diferença não foi evidenciada no terceiro e sexto meses.

\section{Astigmatismo}

A análise da indução de astigmatismo em 4269 olhos submetidos à ablação esférica para correção da miopia mostrou que $1,9 \%$ dos olhos evoluíram com indução de cilindro maior que 0,75 dioptrias. Este número chega a $11,1 \%$ quando se utiliza análise vetorial. Os autores sugerem algumas possibilidades intra e pós-operatórias para justificar esta indução de astigmatismo. Ablação descentrada, alteração na fluência do laser, cicatrização irregular, hiperplasia epitelial e deposição irregular de glicosaminoglicanos. Este estudo concluiu que o astigmatismo induzido é maior quanto maior for o astigmatismo pré-operatório e o equivalente esférico tra$\operatorname{tado}^{(8)}$.

Em nosso estudo não foi observada diferença entre o astigmatismo pré e pós-operatório nos dois grupos. Ressaltamos que não foi realizada análise vetorial nesta avaliação, considerando-se o a diferença entre astigmatismo obtido pela refratometria subjetiva na visita pré-operatória e na visita mais recente de cada paciente.

\section{Complicações}

A diminuição da transparência corneana pós-PRK é um 
fenômeno relativamente comum e relaciona-se com o grau de miopia tratada, sendo mais freqüente em correções acima de 6,00 dioptrias ${ }^{(1)}$. Alguns autores já relataram menor incidência de "haze" e menor intensidade, em pacientes tratados com zona de ablação de $6,0 \mathrm{~mm}$ quando comparado com zona de ablação de 5,0 mm ou múltiplas zonas ${ }^{(7)}$. Na última visita, cinco olhos $(13 \%)$ do presente estudo apresentavam "haze". Apenas um paciente do grupo MZ com "haze" grau I evoluiu com perda de uma linha da acuidade visual corrigida por óculos. Neste mesmo paciente, o "haze" referido, foi observado no décimo segundo mês de pós-operatório. A ocorrência de "haze" tardio em pacientes com equivalente esférico variando de $-4,25$ a $-16,00 \mathrm{D}$ já foi relatada anteriormente ${ }^{(9)}$.

No presente estudo não foi observado padrão topográfico de ilha central em qualquer um dos olhos tratados. Este fato poderia ser atribuído ao pequeno número de pacientes em cada grupo e, com menor probabilidade ao fato de que a primeira videoceratografia ter sido obtida aos 3 meses de pósoperatório, período em que algumas dessas alterações topográficas poderiam ter desaparecido espontaneamente.

\section{CONCLUSÃO}

Os resultados obtidos utilizando-se dois perfis de ablação (multizona e zona única) para tratamento de baixas miopias foram análogos no que diz respeito a equivalente esférico final, indução de astigmatismo e incidência de complicações. Foi observada uma tendência para melhor acuidade visual final no grupo $\mathrm{ZU}$, porém esta diferença não foi considerada importante do ponto de vista clínico. Embora julguemos adequada a utilização de uma amostra maior, o estudo conclui que não foi possível observar diferença entre os dois grupos, podendo-se utilizar qualquer um dos procedimentos no tratamento das miopias até 6 dioptrias.

\section{A B S T RAC T}

Objectives: The present study compares the results obtained with single zone PRK versus multiple zones PRK for treating low myopia. Methods: Nineteen patients presenting spherical equivalent up to 6.00 diopters of myopia, were submitted to PRK using two different approaches. Ramdomly one eye received a $6.0 \mathrm{~mm}$ spherical ablation (SZ) while the fellow eye was treated with two optical zones, $6.0 \mathrm{~mm}$ and $6.5 \mathrm{~mm}$ (MZ).
All the procedures aimed to correct total spherical equivalent. Follow-up was carried out for at least 6 months. Results: The multizone group (MZ) presented a preoperative mean spherical equivalent of -2.87 diopters, ranging from -1.25 to $-4.62 \mathrm{D}$. In the single zone group, the mean spherical equivalent was -2.78 diopters, ranging from -1.37 to $-4.62 \mathrm{D}$. No significant difference was observed between the two groups considering final spherical equivalent $(\mathrm{p}=0.391)$. In the first postoperative month, $84 \%$ of the patients in the MZ group and $95 \%$ in the SZ group presented uncorrected visual acuity equal to or better than $20 / 40$. No difference was detected regarding time necessary to achieve best uncorrected vision. Conclusion: Results obtained using different ablation profiles were similar in regard to spherical equivalent, induced astigmatism and complications. A better final uncorrected visual acuity trend was observed in the SZ group but it was not considered clinically significant. We believe that any of the evaluated approaches are valued methods for treating low myopia.

Keywords: Keratectomy; Photorefractive; Excimer laser; Myopia/Surgery

\section{REFERÊNCIAS}

1. Seiler T, McDonnell PJ. Excimer laser photorefractive keratectomy. Surv Ophthalmol 1995;40:89-118.

2. Hersh PS, Stulting RD, Steinert RF, Waring GO, Thompsom KP, O'Connel M, et al. Results of phase III excimer laser photorefractive keratectomy for myopia. The Summit PRK Study Group. Ophthalmology 1997; 104:1535-53.

3. Gartry DS, Kerr Muir MG, Marshall J. Photorefractive keratectomy with an argon fluoride excimer laser: a clinical study. Refract Corneal Surg 1991;7: 420-35.

4. Hamberg-Nystrum H, Fagerholm P, Tengroth B, Sjuholm C. Thirty-six month follow-up of excimer laser photorefractive keratectomy for myopia. Ophthalmic Surg Lasers 1996;27(5 suppl):S418-20.

5. Taylor HR, McCarty CA, Aldred GF. Predictability of excimer laser treatment of myopia. Arch Ophthalmol 1996;114:248-51.

6. Carones F, Brancato R, Morico A, Venturi E, Gobbi PG. Evaluation of three different approaches to perform excimer laser photorefractive keratectomy for myopia. Ophthalmic Surg Lasers 1996;27(5 suppl):S458-65.

7. Corbett MC, Verma S, O'Brart DP, Oliver KM, Heacock G, Marshall J. Effect of ablation profile on wound healing and visual performance 1 year after excimer laser photorefractive keratectomy. Br J Ophthalmol 1996;80:224-34.

8. Shah S, Chatterjee A, Doyle SJ, Bessant DA. Astigmatism induced by spherical photorefractive keratectomy corrections. Ophthalmology 1997;104: 1317-20.

9. Lipshitz I, Loewenstein A, Varssano D, Lazar M. Late onset corneal haze after photorefractive keratectomy for moderate and high myopia. Ophthalmology 1997;104:369-73.

\section{ABO ELETRÔNICO}

\section{Novo site}

fttp://www.abonet.com.br 